\title{
Simplicity of Beliefs and Delay Tactics in a Concession Game
}

\author{
Ran Spiegler* \\ School of Economics \\ Tel Aviv University \\ Tel Aviv 69978, Israel
}

February 19, 2003

\begin{abstract}
A bstract
I explore the idea of simplicity as a belief-selection criterion in games. A pair of strategies in finite-automata representation $\left(\mathrm{s}_{1}, \mathrm{~s}_{2}\right)$ is a "Simple Nash Equilibrium" (SINE) if: (1) $S_{j}$ is a best-reply to $\mathrm{S}_{\mathbf{i}} ;(2)$ every automaton for player $\mathbf{j}$, which generates the same path as $\mathbf{S}_{\mathbf{j}}$ (given $\mathbf{S}_{\mathbf{i}}$ ), has at least as many states as $\mathbf{S}_{\mathbf{j}}$. I apply SINE to a bilateral concession game and show that it captures an aspect of bargaining behavior: players employ delay tactics in order to justify their concessions. Delay tactics are mutually reinforcing, and this may prevent players from reaching an interior agreement.
\end{abstract}

*I am deeply grateful to the Institute for Advanced Study at Princeton for its stimulating hospitality. I also thank Eddie Dekel, Kfir Eliaz, Michele Piccione, Ariel Rubinstein, an associate editor and a referee for helpful comments. 


\section{Introduction}

The idea that complexity considerations may affect players' choice of strategy has become entrenched in the game-theoretic literature, following Rubinstein (1986), Abreu and Rubinstein (1988), Banks and Sundaram (1990), Piccione (1992), Chatterjee and Sabourian (2000) and others. The assumption underlying these works is that complex strategies are more costly to implement than simple strategies.

By comparison, the analogous idea of complexity as a criterion for belief selection in games has been virtually unexplored. Preference for simple theories is of course an ancient philosophical notion. Usually it is applied to the exalted realm of scientific inquiry, but there is no reason why it should not be relevant to the more mundane business of playing games. One way to implement this idea in the context of two-person games is to assume that among the entire set of beliefs that are consistent with a player's observed behavior, only the simplest ones will be considered by his opponent.

Simplicity is a particularly natural belief-selection criterion when player have justify his behavior to other people. Imagine a "post-mortem" that takes place at the end of the game. Each player is required to explain his behavior to his own audience, which has observed the play path but does not know the opponent's strategy. The explanation consists of a belief i.e., a strategy ascribed to the opponent - and an explanation of the player's response to this belief. When arguments based on common knowledge of rationality are incapable of selecting beliefs, simplicity appears to be a natural selection criterion.

In order to capture this consideration, I introduce a solution concept, called Simple Nash Equilibrium (SINE), for two-person, complete-information games. Restrict attention to pure strategies having a machine (finite automata) representation. A machine profile $\left(s_{1}, s_{2}\right)$ is a SINE if: (1) $s_{i}$ is a best-reply to $s_{\mathrm{j}}$; and (2) $s_{\mathrm{j}}$ is among the least complex strategies for player $j$ that are consistent with the play path induced by $\left(s_{1}, s_{2}\right)$. As has become customary in the literature, the complexity of a machine is quantified by the number its states.

In this paper, I apply SINE to the following game. Two players move alternately. Each player at his turn decides whether or not to make a concession. The game is terminated in agreement if the cumulative number of

concessions reaches some $N>1$. I also study an infinite-horizon version of the game. Players (weakly) prefer any agreement to disagreement. Condi- 
tional on agreement, they strictly prefer to make fewer concessions. I abstract from time preferences; players care only about the concession ratio. In the game's infinite-horizon version, each player tries to maximize the limit proportion of concessions made by his opponent. I refer to this interaction as a concession game.

The concession game is quite uninteresting under Nash and subgame perfect equilibrium: every agreement can be sustained in equilibrium. In contrast, the concept of SINE can lead to drastic reduction in the set of equilibria. In Section 3, I analyze the simple case of $N=2$ as an illustrative exercise and show that there exists no SINE that sustains the interior agreement. In Section 4, I provide two characterization results for the infinite-horizon case. First, under a mild condition, which is closely linked to the "discount robustness" condition due to Kalai and Stanford (1988), no interior agreement is sustainable by a cyclic SINE. Second, if players themselves had a preference for simple (consistent) beliefs, any cyclic SINE which sustains an interior agreement, would be unstable w.r.t this preference. Thus, a taste for simple beliefs, which is shared by the players and their audiences alike, destabilizes any cyclic Nash equilibrium that sustains an interior agreement.

I analyze the case of $2<N<\infty$ in Section 5. This case is more complex to analyze. Therefore, I impose some structure on equilibrium machines: if a state $q$ can be reached from another state $r$, then $r$ cannot be reached from $q$. I refer to machines that satisfy this condition as "linear". This restriction is innocuous under Nash equilibrium: every agreement can be sustained in Nash equilibrium with linear machines. In contrast, there exists no SINE with linear machines that sustains an interior agreement.

The main point of these results is that the pressure to justify concessions can destabilize interior agreements. In SINE, a Player justifies his strategy as a best-reply to a simple, consistent belief of his opponent's strategy. To achieve this goal, the player may have to delay his concessions, and the required amount of delay is increasing in the amount of delay exerted by this opponent. Roughly speaking, if player $i$ delays one of his concessions by $K$ periods, then player $j$ will have to delay his concessions by $K+1$ periods. Thus, the "delay tactics" that result from the pressure to justify concessions are mutually reinforcing. Ultimately, they may prevent the players from reaching an interior agreement.

In general, I believe that the idea of simplicity as a belief-selection criterion can be instrumental in understanding the phenomenon of delay in bargaining interactions. Developing this idea is left for future work. 


\section{Related literature}

This paper is linked to several strands in the literature. The strongest link is with Abreu and Rubinstein (1988). Complexity considerations play subtly different roles in the two papers. A detailed comparison is conducted in Section 6.

The idea of simplicity as a belief-selection criterion has been studied in two recent works. SINE is a special case of the concept of Equilibrium in Justifiable Strategies (EJS), developed in Spiegler (2002). Whereas SINE requires players to optimize against simple consistent beliefs, EJS allows them to depart from best-replying. The concept of EJS will be introduced in Section 6, in the context of concession games with costly delay.

Eliaz (2001) constructs a related solution concept, called "Nash Equilibrium with Stable Forecasts" (ESF). A pair of machines $\left(s_{1}, s_{2}\right)$ is an ESF if: (1) $s_{\mathrm{i}}$ is a best-reply to $s_{\mathrm{j}} ;(2)$ there exists no $s_{\mathrm{j}}^{\prime} \in S_{\mathrm{j}}$ that is simpler than $s_{\mathrm{j}}$, such that $s_{\mathrm{i}}$ is a best-reply to $s_{\mathrm{j}}^{\prime}$. In other words, $s_{\mathrm{j}}$ must be the simplest belief that rationalizes $s_{\mathrm{i}}$. (However, the destabilizing belief $s_{\mathrm{j}}^{\prime}$ need not be consistent with the play path.) Thus, in SINE, $s_{\mathrm{j}}$ is not a stable belief if there is a simpler belief that is consistent with player $j$ 's behavior along the play path, whereas in ESF, $s_{\mathrm{j}}$ is not a stable belief if there is a simpler belief that rationalizes $s_{-\mathrm{j}}$.

Finally, Maenner (2001) uses simplicity as belief-selection criterion within a learning procedure, rather than for equilibrium refinement purposes.

\section{The Model}

Two players play the following complete-information, multi-period game, dubbed "the concession game". Player 1 moves at odd periods and player 2 moves at even periods. Each player at his turn decides between two actions, "concede" $(C)$ and "do not concede" $(D)$. I sometimes refer to $C$ and $D$ as "soft" and "tough". The action taken at period $k$ of some play path is denoted $a^{\mathrm{k}} \in\{C, D\}$.

Let $N>1$ be some finite integer. The case of infinite $N$ is considered in Section 4. The set of terminal histories (play paths) consists of agreement paths, in which the total number of concessions is equal to $N$, and disagreement paths, in which the total number of concessions is smaller than $N$. In agreement paths, $x_{1} \in\left\{0, \frac{1}{N}, \frac{2}{N}, \ldots, 1\right\}$ is the concession ratio in the game - 
i.e., the proportion of player 2's concessions out of the total number of concessions. In disagreement paths, $x_{1}=x_{2}=0$. Player $j$ 's sole objective is to maximize $x_{\mathrm{j}}$. I abstract from time preferences: players are indifferent to delay. The case of costly delay is dealt with in Section 6 .

My favorite interpretation of the concession game is as a model of bargaining over an agenda consisting of $N$ binary issues. The agenda is fixed: players have to resolve one issue before they can proceed to the next issue.

When discussing strategies, I will restrict attention to pure strategies that can be represented by a machine $\left(Q, q^{0}, f, \tau\right)$, where $Q$ is a finite set of states; $q^{0}$ is the initial state; $f: Q \rightarrow\{C, D\}$ is an output function, which specifies the action taken by the player when he is in state $q \in Q$; and $\tau: Q \times\{C, D\} \rightarrow Q$ is a transition function, which specifies the state to which the machine switches from state $q \in Q$ when the opponent plays $a \in\{C, D\}$ against $q$.

A bit of extra terminology and notation will be useful:

- When $f(q)=a, q$ is called an a-state.

- For any $q, r \in Q$, we say that $r$ can be reached from $q$ if there exists a sequence of states $q^{1}, \ldots, q^{\mathrm{m}} \in Q$ and a sequence of actions $a^{1}, \ldots, a^{\mathrm{m}-1} \in$ $\{C, D\}$, such that $q^{1}=q, q^{\mathrm{m}}=r$, and for every $k=1, \ldots, m-1$, $\tau\left(q^{\mathrm{k}}, a^{\mathrm{k}}\right)=q^{\mathrm{k}+1}$.

- The path induced by a machine profile $\left(s_{1}, s_{2}\right)$ is denoted $z\left(s_{1}, s_{2}\right)$.

- The machine state of the player who moves at period $k$ in $z\left(s_{1}, s_{2}\right)$ is denoted $p(k)$. Note that $p(1)=q_{1}^{0}$ and $p(2)=\tau_{2}\left(q_{2}^{0}, a^{1}\right)$.

- A state $q \in Q_{\mathrm{i}}$ is visited in $z\left(s_{1}, s_{2}\right)$ if $p(t)=q$ for some period $t$ in $z\left(s_{1}, s_{2}\right)$.

- A machine profile $\left(s_{1}, s_{2}\right)$ sustains an interior agreement if each player makes at least one concession along the induced path.

The restriction to machines entails no loss of generality: every play path in the concession game can be generated by a pair of machines. In particular, 
every agreement $x_{1} \in\left\{0, \frac{1}{N}, \ldots, 1\right\}$ is sustainable by some pair of strategies with a machine representation, which constitutes a Nash equilibrium. ${ }^{1}$

The machine representation enables us to formalize complexity considerations. Following common practice, the complexity of a machine is measured by number of its states. A machine $b$ for player $j$ is consistent with $\left(s_{1}, s_{2}\right)$ if $z\left(s_{\mathrm{i}}, b\right)=z\left(s_{1}, s_{2}\right)$. Let $B_{\mathrm{j}}\left(s_{1}, s_{2}\right)$ be the set of simplest consistent machines for player $j$, given $z\left(s_{1}, s_{2}\right)$. That is, $b \in B_{\mathrm{j}}\left(s_{1}, s_{2}\right)$ if $b$ is consistent with $z\left(s_{1}, s_{2}\right)$ and every other machine for player $j$, which is consistent with $z\left(s_{1}, s_{2}\right)$, has at least as many states as $b$.

Definition 1 A pair of machines $\left(s_{1}, s_{2}\right)$ is a Simple Nash Equilibrium (SINE) if for every $j=1,2, i \neq j$ :

1. $s_{\mathrm{i}}$ is a best-reply to $s_{\mathrm{j}}$.

2. $s_{\mathrm{j}} \in B_{\mathrm{j}}\left(s_{1}, s_{2}\right)$

I adhere to the tradition of interpreting equilibrium strategies as beliefs i.e., $s_{\mathrm{j}}$ is player $i$ 's belief of player $j$ 's strategy, where the belief is cast in the form of a machine. Under this interpretation, the concept of SINE captures the idea that in equilibrium, it is not enough for player $i$ 's belief to be correct - it also has to be simple. There should exist no belief of player $j$ 's strategy, which is simpler than $s_{\mathrm{j}}$ and consistent with $z\left(s_{1}, s_{2}\right)$.

The concession ratios $x_{1}=1$ and $x_{1}=0$ are SINE-sustainable for every $N$. First, the strategies "always $D$ " and "always $C$ " are best-replies to each other, hence they satisfy the first part of Definition 1. Second, both strategies have a single-state machine representation, hence they satisfy the second part of Definition 1. Of course, we are more interested in the SINEsustainability of interior agreements. This will be the subject-matter of the following sections.

\footnotetext{
${ }^{1}$ E.g., player j's concessions can be rationalized by a "grim" threat by his opponent namely, that player $\mathrm{i}$ will stop making further concessions unless player $\mathrm{j}$ concedes first. The same holds for subgame perfect equilibrium, under a modification of the machine formalism, which allows players to condition on their own past moves.
} 


\section{The Case of $N=2$}

Studying the simple case of $N=2$ first will be useful for illustrating the effect of simplicity as a belief-selection criterion on the sustainability of interior agreements in the concession game.

Proposition 1 There exists no SINE that sustains the agreement $x_{1}=\frac{1}{2}$.

P roof. Assume the contrary and let $\left(s_{1}, s_{2}\right)$ be a SINE sustaining the agreement $x_{1}=\frac{1}{2}$. Each player plays $C$ exactly once along the equilibrium path. If player $i$ never plays $D$ along the play path, then $B_{\mathrm{i}}\left(s_{1}, s_{2}\right)$ consists of a single machine, consisting of a single $C$-state. Against this machine, player $j$ 's concession is sub-optimal, a contradiction. Therefore, each player has to play $D$ at least once in $z\left(s_{1}, s_{2}\right)$. It follows that each player's machine contains at least one $D$-state and one $C$-state.

Let $K_{\mathrm{i}} \in\{0,1,2, \ldots\}$ denote the number of consecutive periods, in which both players play $D$ just before player $i$ makes his concession. (E.g., in the play path given in the example below this proof, $K_{1}=K_{2}=4$.) W.l.o.g., $K_{\mathrm{i}} \geq K_{\mathrm{j}}$. During these $K_{\mathrm{i}}$ periods, no state $q \in Q_{\mathrm{i}}$ can be visited more than once. Assume the contrary. Then, there exists a sequence of states $\left(q^{1}, \ldots, q^{\mathrm{m}}\right)$ in $Q_{\mathrm{i}}$, such that: $q^{1}=q^{\mathrm{m}}=q$, and $f_{\mathrm{i}}\left(q^{\mathrm{k}}\right)=D$ and $\tau_{\mathrm{i}}\left(q^{\mathrm{k}}, D\right)=q^{\mathrm{k}+1}$ for every $k=1, \ldots, m-1$. However, this contradicts the fact, evident from the play path, that if player $j$ starts playing $D$ repeatedly when player $i$ 's machine is in $q$, player $i$ 's machine eventually reaches a $C$-state. It follows that no state in $Q_{\mathrm{i}}$ is visited more than once during the $K_{\mathrm{i}}$ periods that immediately precede player $i$ 's concession. Let $M$ denote the number of times that player $i$ plays $D$ during these $K_{\mathrm{i}}$ periods. ( $M$ is equal to $\frac{\mathrm{K}_{\mathrm{i}}}{2}$, rounded either up or down.) Thus, player $i$ 's machine contains at least one $C$-state and $M$ $D$-states, such that $\left|Q_{\mathrm{i}}\right| \geq M+1$.

Let us establish that every member in $B_{\mathrm{i}}\left(s_{1}, s_{2}\right)$ contains exactly $M+1$ states. We shall do this by constructing a machine for player $i$ consisting of $M+1$ states, which is consistent with $z\left(s_{1}, s_{2}\right)$. Let us carry out the construction for the case of $i=2$. (The construction for the case of $i=1$ is virtually identical and therefore omitted, for the sake of brevity.) Let $Q_{2}=\left\{q^{0}, \ldots, q^{\mathrm{M}}\right\}$; the initial state is $q^{0}$; let $f_{2}\left(q^{0}\right)=C$ and $f_{2}\left(q^{\mathrm{k}}\right)=D$ for every $k \neq 0$; finally, let $\tau_{2}\left(q^{\mathrm{k}}, D\right)=q^{\mathrm{k}+1 \bmod \mathrm{M}+1}$ and $\tau_{2}\left(q^{\mathrm{k}}, C\right)=q^{1}$ for every 
$k=0, \ldots, M$. This machine is consistent with $z\left(s_{1}, s_{2}\right)$ because $K_{2} \geq K_{1}{ }^{2}$

It remains to show that facing any machine for player $i$, which is consistent with $z\left(s_{1}, s_{2}\right)$ and contains exactly $M+1$ states, player $j$ 's concession is suboptimal. If $\left|Q_{\mathrm{i}}\right|=M+1$, then every $D$-state in player $i$ 's machine is visited during the $K_{\mathrm{i}}$ periods that immediately precede his concession. According to the play path, if player $j$ starts playing $D$ repeatedly when player $i$ 's machine is in any of these $D$-states, player $i$ eventually makes a concession. Thus, given player $i$ 's machine, player $j$ could have enforced the outcome $x_{\mathrm{j}}=1$ instead of $x_{\mathrm{j}}=\frac{1}{2}$, hence $s_{\mathrm{i}}$ is not a best reply to $s_{\mathrm{j}}$, a contradiction.

To illustrate the reasoning involved in this result, consider the following play path:

$\begin{array}{ccccccccccc}\text { Period } & 1 & 2 & 3 & 4 & 5 & 6 & 7 & 8 & 9 & 10 \\ \text { Player 1 } & D & & D & & C & & D & & D & \\ \text { Player 2 } & & D & & D & & D & & D & & C\end{array}$

Player 1 needs to justify his concession at period 5 as part of a best reply to player 2's strategy. One way to do this is to argue that if he had not made that first concession, player 2 would never have conceded and there would have been no agreement. Figure 1 represents a belief of player 2's strategy, which is consistent with such a justification:

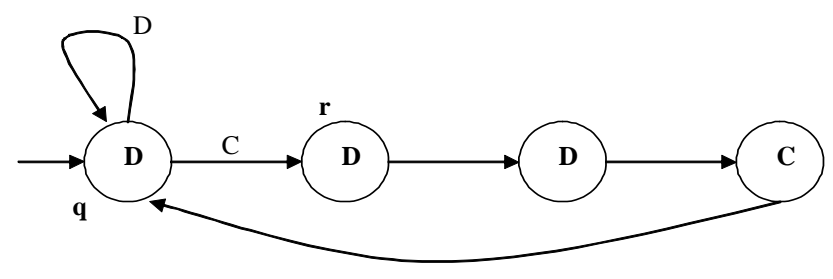

Figure 1

\footnotetext{
${ }^{2}$ E.g., in the play path given in the example below this proof, $M=2$. The reader can verify that the machine given by Figure 2 matches the general construction just given.
} 
However, $s_{2}$ is not a simplest consistent belief of player 2's strategy, given the play path: the machine given by Figure 2 consists of fewer states:

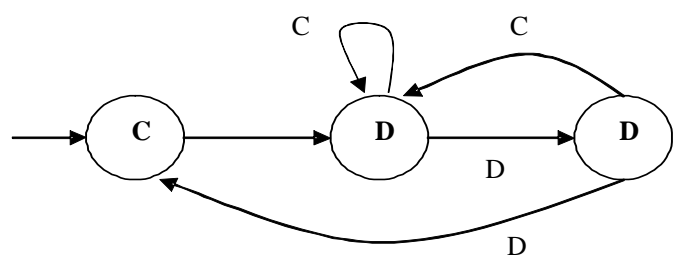

Figure 2

Note that if player 1 faced the machine represented by Figure 2, it would not be optimal for him to make any concession. In general, as long as player 1 fails to delay his concession longer than player 2 delays his own concession, no simplest consistent machine for player 2 can justify player 1's concession. The only way for player 1 to justify his concession is by delaying it longer than player 2 does. Only by doing so, will he be able to justify his concession as optimal against a simplest consistent belief of player 2's strategy. Clearly, this kind of delay tactics is mutually reinforcing. The more player 1 delays his concession, the more player 2 will need to delay his own concession in order to justify it. As a result, the interior agreement cannot be sustained.

The complexity considerations leading to the players' delay tactics capture the following intuition. The longer the delay between two concessions, the weaker the perceived causal link between them. In other words, as player 1's concession becomes more distant in time than player 2's concession, the claim that former was the cause of the latter is less persuasive. It is not player 1's concession, but rather his subsequent tough play that appears to have caused player 2 to concede. In order to persuade his audience that his concession was necessary, player 1 needs to make the delay between the two concessions appear short relative to the total duration of the game. Hence player 1's motive to delay his concession longer than player 2 delays his own concession. 


\section{An Infinite-Horizon Concession Game}

In this section, I study an infinite-horizon version of the concession game. This is a bargaining model, in which the two parties fight over an indefinite number of binary issues. This description especially fits situations of relational bargaining. The lack of a finite horizon allows us to obtain rich results because there are no end-game effects that complicate the analysis.

Of course, we need to redefine the payoffs. Given a machine profile $\left(s_{1}, s_{2}\right)$, define $X_{\mathrm{i}}(t)$ as the cumulative number of concessions made by player $j$ by period $t$. Then, a disagreement path is a path, for which:

$$
\lim _{t \rightarrow \infty} \frac{X_{\mathrm{i}}(t)}{t}=0
$$

for both $i=1,2$. For agreement paths, the limit is strictly positive for at least one player. Then:

$$
x_{\mathrm{i}}=\lim _{\mathrm{t} \rightarrow \infty} \frac{X_{\mathrm{j}}(t)}{X_{\mathrm{i}}(t)+X_{\mathrm{j}}(t)}
$$

These limits are well-defined because any profile of finite automata eventually enters a cycle. The cycle in a disagreement path consists of nothing but $D$ 's. The cycle of an agreement path contains at least one $C$. We will say that an agreement path induces an interior agreement if $x_{\mathrm{i}}>0$ for both $i=1,2$. Note that in any agreement path, $x_{1}+x_{2}=1$. Assume that players care only about the limit concession ratio. That is, player $j$ 's sole objective is to maximize $x_{\mathrm{j}}$.

A pair of machines $\left(s_{1}, s_{2}\right)$ is cyclic if there exists an integer $L$, such that for every player $j$ and every period $t \geq 1, p_{\mathrm{j}}(t)=p_{\mathrm{j}}(t+L)$. That is, the cyclic phase of the play path begins right at the beginning of the game. One motivation for studying cyclic equilibria is that the player's audience may have bounded recall. The audience cannot survey the entire play path, but only some backward truncation of it.

Given a machine $s_{\mathrm{j}}$ for player $j$, define $V_{\mathrm{i}}(q, a)$ as the maximal payoff that player $i$ can obtain using a strategy that always plays $a \in\{C, D\}$ when $s_{\mathrm{i}}$ is in $q \in Q_{\mathrm{j}}$. For example, when player 2's machine is given by Figure $1, V_{1}(q, D)=0, V_{1}(q, C)=\frac{1}{2}$, whereas $V_{1}\left(q^{\prime}, D\right)=\frac{1}{2}$ and $V_{1}\left(q^{\prime}, C\right)=\frac{1}{3}$ for every other state $q^{\prime} \in Q_{2}$. 
Definition 2 A pair of machines $\left(s_{1}, s_{2}\right)$ satisfies "No Redundant Threats" (NRT) if for every $i=1,2, j \neq i$, and every $q \in Q_{\mathrm{j}}, \tau_{\mathrm{j}}(q, C) \neq \tau_{\mathrm{j}}(q, D)$ implies $V_{\mathrm{i}}(q, C) \neq V_{\mathrm{i}}(q, D)$.

The NRT property means that if playing $C$ and $D$ against a state $q$ leads to different continuations, then the player cannot be indifferent between always playing $C$ against $q$ and always playing $D$ against $q$. That is, all the threats in players' machines have to be payoff-relevant. The machines given by Figures 1 and 2 satisfy the NRT property, whereas the machine given by Figure 3 violates it: playing $C$ or $D$ against $q$ leads to different continuations, yet $V(q, C)=V(q, D)=\frac{1}{2} .^{3}$

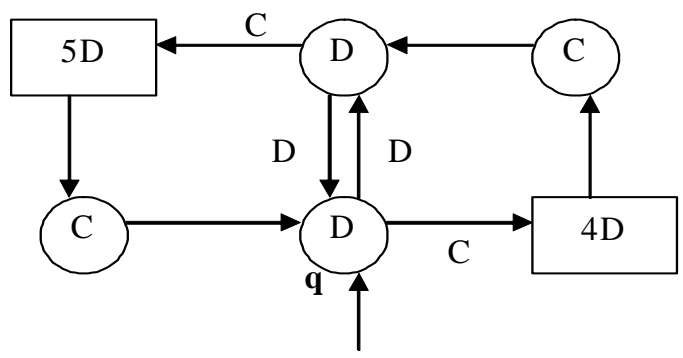

Figure 3

In a repeated game with discounting, the NRT property is equivalent to the "discount robustness" condition due to Kalai and Stanford (1988). Basically, NRT and discount robustness are conditions that can be used in order to rule out equilibria that crucially rely on ties.

The NRT property is innocuous under Nash or subgame perfect equilibrium. Every concession ratio $x_{1} \in[0,1]$ in the infinite-horizon concession game can be approximated by a Nash equilibrium that is implemented by a machine profile satisfying NRT.

\footnotetext{
${ }^{3}$ The box $k D(k=4,5)$ means that $k D$-states machine are serially connected by a constant transition - i.e., the machine plays $\mathrm{D}$ for $\mathrm{k}$ times in a row, independently of the opponent's moves.
} 
Proposition 2 There exists no cyclic SINE that satisfies the NRT property and sustains an interior agreement.

The reasoning behind this result consists of two stages. The first stage establishes that in any cyclic SINE satisfying NRT, each player makes at least one concession (per cycle), which he justifies by the claim that otherwise, the opponent would have stayed in the same $D$-state indefinitely. In other words, each player plays $C$ at least once against a "stalemate" - i.e., a state $q$ satisfying $f_{\mathrm{j}}(q)=D$ and $\tau_{\mathrm{j}}(q, D)=q$. The second stage is similar to the proof of Proposition 1. Players justify concessions by delaying them. Their delay tactics are mutually reinforcing, such that an interior agreement is unsustainable: there will always be at least one player, who wishes he had delayed his concessions longer.

Once the NRT property is relaxed, it becomes possible to sustain interior agreements in SINE. E.g., when both players play the machine given by Figure 3 (except that the initial state in the machine of one of the players is adjusted), a cyclic SINE is obtained. The cycle is a succession of four chunks of the following form: player 1 makes a concession after $d_{1}$ periods, then player 2 makes a concession after $d_{2}$ periods, where $d_{1}, d_{2} \in\{4,5\}$. The cycle contains all four combinations of $\left(d_{1}, d_{2}\right)$. The players' indifference between delaying concessions by four or five periods allows a relatively irregular cycle to be generated from relatively simple machines.

This SINE has an interesting property. Player 1, say, is able to deviate to another strategy $s_{1}^{\prime}$, which prescribes only $D$ against $q$ and attains the same payoff as $s_{1}$. Several states in $s_{2}$ remain unvisited along $z\left(s_{1}^{\prime}, s_{2}\right)$. Therefore, player 1 can justify $s_{1}^{\prime}$ as a best-reply to a simpler machine for player 2, given in Figure 4:

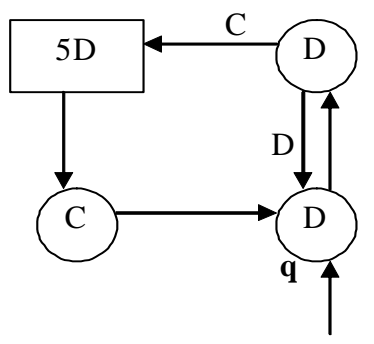

Figure 4 
In other words, player 1 can deviate to a strategy, which he can justify as a best-reply to a belief, which is both simpler than $s_{2}$ and consistent with the path resulting from player 1's deviation. Furthermore, player 1 can achieve this without compromising optimal response to $s_{2}$. This turns out to be a general property.

Proposition 3 Suppose that $\left(s_{1}, s_{2}\right)$ is a cyclic SINE that sustains an interior agreement. Then, there is a player $i$ and a pair of machines, $s_{\mathrm{i}}^{\prime}$ and $s_{\mathrm{j}}^{\prime}$, such that:

1. $s_{\mathrm{j}}^{\prime}$ is a best-reply to $s_{\mathrm{i}}$ and $s_{\mathrm{i}}^{\prime}$.

2. $s_{\mathrm{i}}^{\prime}$ is consistent with $z\left(s_{\mathrm{i}}, s_{\mathrm{j}}^{\prime}\right)$.

3. $s_{\mathrm{i}}^{\prime}$ is simpler than $s_{\mathrm{i}}$.

What is the meaning of this result? Recall that SINE captures the idea that complex beliefs are inadmissible when a player tries to justify his behavior before an audience. We might say that the concept reflects the audience's preference for simple beliefs. Alternatively, we could be interested in situations, in which it is the player himself who prefers to justify his behavior with simple beliefs. One rationale behind such a preference is that complex beliefs have a greater chance of being misunderstood by the player's audience. Given this preference, a player has a motive to deviate if he can keep optimizing against the opponent's true strategy, while reducing the complexity of the (consistent) belief that he uses to rationalize his behavior. ${ }^{4}$

Proposition 3 shows that there is a clash between these two types of preference for simple beliefs: the player's and his audience's. If a cyclic Nash equilibrium sustaining an interior agreement is robust w.r.t the audience's preference for simple consistent beliefs, then it is not robust w.r.t the player's preference for simple consistent beliefs, and vice versa.

In the example that motivates Proposition 3, player 1's deviation clearly satisfies all three conditions of the proposition. When player 1 stops playing

\footnotetext{
${ }^{4}$ In Eliaz (2001), players have a preference for simple beliefs. The difference is that in Eliaz (2001), the simpler belief need not be consistent with the play path: a simpler rationalizing belief is always favored, whether or not it is consistent with the play path.
} 
$C$ against $q$, the whole "right-hand side" of player 2's machine is never visited. Therefore, it is possible to obtain a simpler machine for player 2, which would be consistent with the path induced by player 1's deviation, as Figure 4 illustrates. The main difficulties in proving Proposition 3 arise when such a deviation is unavailable - i.e., when the set of states in player $j$ 's machine, which are visited along the play path, does not change when player $i$ deviates to a strategy that avoids playing $C$ against some state $q \in Q_{\mathrm{j}}$.

Propositions 2 and 3 characterize the set of cyclic SINE in the infinitehorizon concession game. The restriction to cyclic equilibria is important for the results of this section. Typically, manipulating $B_{\mathrm{j}}\left(s_{1}, s_{2}\right)$ has payoffrelevant consequences for player $i$. However, in cyclic equilibria, there is a single exception: if player $i$ changes the number of times that he plays $D$ against a state $q \in Q_{\mathrm{j}}$ satisfying $f_{\mathrm{j}}(q)=D$ and $\tau_{\mathrm{j}}(q, D)=q$, he may affect $B_{\mathrm{j}}\left(s_{1}, s_{2}\right)$ without affecting the limit concession ratio. This feature is crucial for the proofs of Propositions 2 and 3. (In particular, see Claim 3 in the proof of Proposition 2.) In contrast, when $z\left(s_{1}, s_{2}\right)$ has an introductory phase, player $i$ can also use his behavior in that phase to manipulate $B_{\mathrm{j}}\left(s_{1}, s_{2}\right)$, without affecting the limit concession ratio. This makes the characterization of such equilibria considerably more difficult.

\section{The Case of $2<N<\infty$}

In the proof of both Propositions 1 and 2, a crucial property is that player $j$ plays a unique action in equilibrium against $q \in Q_{\mathrm{i}}$, except possibly when $q$ is what I referred to as a "stalemate". This is no longer the case when $2<N<\infty$. For example, suppose that $s_{1}$ contains an absorbing $D$-state, which is reached after $N-k$ concessions have been made. Then, player 2 has to make the $k$ last concessions in the game, but he can intersperse these concessions with a number of $D$ 's, without sacrificing optimality. Contrary to the case of $N=\infty$, an auxiliary condition such as NRT is nonsensical because the function $V(q, \cdot)$ is ill-defined.

The possibility of playing both softly and toughly against states other than stalemates complicates analysis considerably. Therefore, in this section I impose some structure on equilibrium machines. 
Definition 3 A machine $s=\left(Q, q^{0}, f, \tau\right)$ is linear if for every two distinct states $q, r \in Q$, if $r$ can be reached from $q \in Q$, then $q$ cannot be reached from $r$.

Linearity means that the machine contains no cycles. Once a state is "abandoned", it can never be visited again. Note, however, that linearity allows $\tau(q, a)=q$ for some $a \in\{C, D\}$. The restriction to linear machines entails no loss of generality under Nash equilibrium. Every agreement $x \in$ $\left\{0, \frac{1}{N}, \ldots, 1\right\}$ can be sustained by a Nash equilibrium with linear machines. For example, the pair of linear machines given by Figure 5 constitutes a Nash equilibrium for every $N>2$, sustaining the concession ratio $x_{1}=\frac{N-1}{N}$.

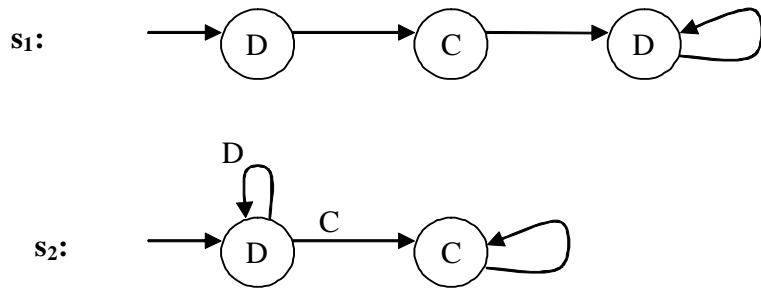

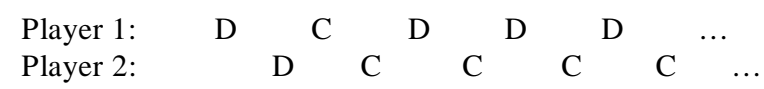

Figure 5

This, however, is not a SINE because $s_{1}$ is not the simplest machine that can generate player 1's behavior. A machine consisting of a single $C$-state $q^{\mathrm{C}}$ and a single $D$-state $q^{\mathrm{D}}$ satisfying $\tau\left(q^{\mathrm{D}}, C\right)=q^{\mathrm{D}}$ and $\tau\left(q^{\mathrm{D}}, D\right)=q^{\mathrm{C}}$ generates the same path.

The chief attraction of linear machines is that they are easy to construct as an explanation for a given play path, because whenever $p_{\mathrm{i}}(t+2) \neq p_{\mathrm{i}}(t)$, $p_{\mathrm{i}}(t)$ is never revisited after period $t$. In other respects, linearity is a strong restriction. In particular, given a particular play path, the simplest consistent linear machine for a player may contain many more states than the simplest consistent machine in the unrestricted domain. 
Proposition 4 There exists no SINE with linear machines that sustains an interior agreement $x_{1} \in\left\{\frac{1}{N}, \ldots, \frac{N-1}{N}\right\}$.

Thus, a class of machines that suffices to sustain any interior agreement under Nash equilibrium turns out to be incapable of sustaining interior agreements under SINE. From a technical point of view, what makes the proof go through is the fact that in linear machines, a state $q \in Q_{\mathrm{i}}$ is visited more than once only if it $\tau_{\mathrm{i}}(q, a)=q$ for some $a \in\{C, D\}$. This is the only kind of states in player $i$ 's machine, against which player $j$ might play more than just one action along the equilibrium path. This allows us to use the same kind of reasoning used in previous results. Other restrictions on the domain of permissible machines leading to the same property would imply the same impossibility result. ${ }^{5}$

As mentioned earlier, linear machines are attractive because of their ease of construction as an explanation for a given play path. Proposition 4 shows that in order to sustain an interior agreement in SINE, the players' machines have to be non-linear. In this respect, if an interior agreement can be sustained in equilibrium, then equilibrium strategies must be hard to construct.

The restriction to linear machines plays the same role in the analysis of SINE in the case of $2<N<\infty$ as the NRT property in the case of $N=\infty$. Each restriction in its case implies the property that player $j$ plays a unique action against any state in player $i$ 's machine, except for states having a particular structure.

\section{Comparison with A breu-R ubinstein (1988)}

The concept of SINE bears a close formal resemblance to the model of Abreu and Rubinstein (1988) - AR henceforth. The cornerstone of AR is the assumption that players prefer simpler strategies. That is, complexity considerations are applied to strategies, rather than beliefs. The special case, in which complexity costs are lexicographically secondary to "regular" payoffs, is of particular relevance to the present paper.

\footnotetext{
${ }^{5}$ Note that when $\mathbf{N}=\infty$, the restriction to linear machines is trivial, as it immediately implies a limit concession ratio of 0 or 1 , independently of equilibrium arguments.
} 
Definition 4 A pair of machines $\left(s_{1}, s_{2}\right)$ is an $\mathrm{AR}$-equilibrium if for every $j=1,2, i \neq j$ :

1. $s_{\mathrm{i}}$ is a best-reply to $s_{\mathrm{j}}$.

2. There exists no machine $s_{\mathrm{i}}^{\prime}$, which is a best-reply to $s_{\mathrm{j}}$ and has fewer states than $s_{\mathrm{i}}$.

\section{Remark 1 Every AR-equilibrium is a SINE.}

The proof is trivial. If there exists no strategy $s_{\mathrm{i}}^{\prime}$, which is simpler than $s_{\mathrm{i}}$ and generates the same payoff for player $i$, given $s_{\mathrm{j}}$, then clearly there exists no strategy $s_{\mathrm{i}}^{\prime}$, which is simpler than $s_{\mathrm{i}}$ and generates the same path as $s_{\mathrm{i}}$, given $s_{\mathrm{j}}$. Remark 1 holds for every game and every measure of complexity.

The concept of $A R$-equilibrium was originally conceived in the context of two-person, infinitely repeated games with discounting. In this class of games, a converse result to Remark 1 holds.

Remark 2 Every SINE in a two-person, infinitely repeated game with discounting, which satisfies NRT, is also an $\mathrm{AR}$-equilibrium.

Once again, the proof is simple. Let $\left(s_{1}, s_{2}\right)$ be a SINE. By the discounting assumption, $a$ is played against $q \in Q_{\mathrm{j}}$ if and only if $a \in \arg \max V_{\mathrm{i}}(q, \cdot)$. By NRT, $\arg \max V_{\mathrm{i}}(q, \cdot)$ is a singleton for every $q \in Q_{\mathrm{j}}$. Therefore, no other path generates the same payoff for player $i$ as $z\left(s_{1}, s_{2}\right)$, given $s_{\mathbf{j}}$. By definition of SINE, it is impossible to generate $z\left(s_{1}, s_{2}\right)$ with simpler machines. It follows that $\left(s_{1}, s_{2}\right)$ is an $\mathbf{A} \mathbf{R}$-equilibrium.

In the concession game, Remark 2 is not true. For instance, consider the following pair of machines. Player 1's machine $s_{1}$ consists of a single $D$-state. Player 2's machine $s_{2}$ is: $Q_{2}=\left\{q^{\mathrm{C}}, q^{\mathrm{D}}\right\}, q_{2}^{0}=q^{\mathrm{C}}, f_{2}\left(q^{\mathrm{C}}\right)=C$, $f_{2}\left(q^{\mathrm{D}}\right)=D, \tau_{2}\left(q^{\mathrm{C}}, \cdot\right)=q^{\mathrm{D}}, \tau_{2}\left(q^{\mathrm{D}}, \cdot\right)=q^{\mathrm{C}}$. On one hand, $\left(s_{1}, s_{2}\right)$ is a SINE. Along $z\left(s_{1}, s_{2}\right)$, player 1 always plays $D$, whereas player 2 plays both $C$ and $D$. Since $s_{1}$ is a single-state machine, $B_{1}\left(s_{1}, s_{2}\right)=\left\{s_{1}\right\}$. As to player 2 , his machine consists of a single $C$-state and a single $D$-state, hence it is impossible to reduce the number of states in his machine without violating consistency with the play path. On the other hand, $\left(s_{1}, s_{2}\right)$ is not an ARequilibrium. A machine for player 2 , which consists of a single $C$-state has 
fewer states than $s_{2}$, yet it induced the same concession ratio. Thus, SINE and $A R$-equilibrium diverge in the concession game.

An immediate implication of Remark 1 is that any impossibility result proved for SINE carries over to AR-equilibrium as well. At least in the case of $N=\infty$, a general impossibility result that interior agreements cannot be sustained in AR-equilibrium is provable, using methods borrowed from Piccione (1992) and Piccione and Rubinstein (1993).

However, it should be emphasized that although the results are similar, the reasoning that leads to them is quite different under the two concepts. Put succinctly, SINE implies a motive to delay concessions, whereas ARequilibrium implies a motive to hasten concessions. Consider the case of $N=2$, for instance. In Section 3, we saw that when player 2 plays the machine given by Figure 1, SINE implies that player 1 delays his concession by at least three periods. In contrast, $A R$-equilibrium implies that player 1 should not delay his concession at all, but rather play $C$ at period 3 , as soon as player 2 reaches state $q$, as this would save unnecessary $D$-states in player 1 's machine.

As a further illustration, consider a simultaneous-move version of the infinite-horizon concession game. Suppose that both players play the machine given by Figure 1, with one modification: player 2's initial state is $r$ instead of $q$. This machine profile is a SINE, yet it does not constitute an AR equilibrium. This example demonstrates not only the difference between the two concepts, but also the role of alternating moves in this paper.

To sum up, the concepts of SINE and A R -equilibrium are formally similar. However, in the concession game they imply different kinds of reasoning. The need to optimize with the simplest possible machine impels players to avoid delay, whereas the need to render the opponent's machine a simple, consistent belief impels them to exert delay.

\section{Concluding R emarks}

Participants in bargaining interactions often want to justify the concessions that they make along the way. One common way of ensuring the justifiability of a concession is to delay it. After a sufficient amount of delay, the concession appears to be necessary in order to break away from a stalemate. This intuition is captured in this paper: delay tactics emerge as an implication

of SINE. Players resort to delay tactics because it helps them establish the 
opponent's strategy as a simple, consistent belief. The mutually reinforcing aspect of this kind of delay tactics may prevent players from reaching an interior agreement. This is one of the main points of this paper: applying complexity considerations to beliefs can be useful in understanding bargaining behavior.

The reader should be reminded that delay does not emerge as an equilibrium phenomenon in the characterization results. What these results show is that interior agreements may be unstable because of delay tactics originating from justifiability considerations - not that delay itself is stable. It certainly cannot be said that this paper "explains" the phenomenon of delay in bargaining.

One element of bargaining, which is absent from the concession game is time preferences: players are assumed to be indifferent to delay. Alternatively, consider the case of $N=2$, and suppose that players' payoffs are strictly decreasing in the duration of the game. Then, the following simple result holds:

Remark 3 Suppose that given $s_{\mathrm{i}}$, the maximal concession ratio that player $j$ can attain is $x_{\mathrm{j}}=\frac{1}{2}$. Then, there exists no machine $s_{\mathrm{j}}$ for player $j$, such that $s_{\mathrm{j}}$ is a best-reply to $s_{\mathrm{i}}$ and $s_{\mathrm{i}} \in B_{\mathrm{i}}\left(s_{1}, s_{2}\right)$.

The proof is simple. If player $j$ has to make a concession in order to squeeze a concession from player $i$, this must be due to some threat of player $i$. When delay is costly, player $j$ cannot realize this threat without sacrificing optimality. But if player $j$ does not realize the threat, this means that certain states in $Q_{\mathrm{i}}$ are not visited in the play path. Hence, $s_{\mathrm{i}}$ is not a simplest consistent machine for player $i$.

It follows that when delay is costly, the impossibility of sustaining the interior agreement in SINE holds trivially. The tension between simple beliefs and optimal response is not an equilibrium phenomenon: it already arises at the level of individual behavior.

In Spiegler (2002), I argue that if sacrificing optimality is necessary for the player's belief to be convincing in the eyes of his audience, then a proper notion of justifiability should take this into account. In Spiegler (2002), the game's "post-mortem" is viewed as a debate, in which arguments and counter arguments are exchanged between the player and his audience. 
Definition 5 A machine $s_{\mathrm{i}}$ is justifiable against $s_{\mathrm{j}}$ if:

1. $s_{\mathrm{j}} \in B_{\mathrm{j}}\left(s_{\mathrm{i}}, s_{\mathrm{j}}\right)$

2. For every profitable deviation $s_{\mathrm{i}}^{\prime}$ for player $i$, and every belief $b \in$ $B_{\mathrm{j}}\left(s_{\mathrm{i}}^{\prime}, s_{\mathrm{j}}\right), s_{\mathrm{i}}$ does better than $s_{\mathrm{i}}^{\prime}$ against $b$.

Definition 5 captures the following "post-mortem". Player $i$ 's audience accepts only a simple, consistent belief of player $j$ 's strategy. Suppose that $s_{\mathrm{j}}$ is an admissible belief, but $s_{\mathrm{j}}$ is not an optimal response to $s_{\mathrm{j}}$. Player $i$ 's audience raises the criticism that an alternative machine $s_{\mathrm{i}}^{\prime}$ would have done better than $s_{\mathrm{i}}$ against $s_{\mathrm{j}}$. Suppose that player $i$ can counter-argue that had he played $s_{\mathrm{i}}^{\prime}$, he would have been subjected to the same kind of criticism, only in the opposite direction: $s_{\mathrm{i}}$ is better than $s_{\mathrm{i}}^{\prime}$ against every admissible belief given the path induced by $\left(s_{\mathrm{i}}^{\prime}, s_{\mathrm{j}}\right)$. This is a "smashing" counter-argument because it shows that the audience's criticism is self-defeating: if the player had accepted the criticism, he would have still been exposed to it. If player $i$ can counter-argue in this way against every criticism of his audience, his machine is justifiable against $s_{\mathrm{j}}$. We say that $\left(s_{1}, s_{2}\right)$ is an Equilibrium in Justifiable Strategies (EJS) if $s_{1}$ is justifiable against $s_{2}$, and vice versa. The concept of SINE is a special case of EJS: Every SINE is an EJS.

Justifiability allows a player to play sub-optimally if he can defend his departure from best-replying with the right counter-argument. Turning back to the case of $N=2$, It can be shown that if the cost of delay is sufficiently small and player 2's machine $s_{2}$ is given by Figure 1, there exists a machine $s_{1}$ for player 1 , which is justifiable against $s_{2}$. Player 1 attains justifiability in the same way as in Section 3: he delays his concession for three periods.

No EJS sustains the interior agreement in the case of $N=2$. The proof shares many features with the proof of Proposition 1: players attain justifiability through delay tactics, but such tactics are mutually reinforcing, such that in equilibrium there is always at least one player who wishes he had delayed his concession longer. Working out the fuller implications of justifiability for bargaining behavior under costly delay is left for future research. 


\section{R eferences}

1. Abreu D. and A. Rubinstein (1988). "The Structure of Nash Equilibrium in Repeated Games with Finite Automata." Econometrica 56, 1259-1282.

2. Banks J. and R. Sundaram (1990). "Repeated Games, Finite Automata and Complexity." Games and Economic Behavior 2, 97-117.

3. Chatterjee K. and H. Sabourian (2000). "Multiperson Bargaining and Strategic Complexity." Econometrica 68, 1491-1509.

4. Eliaz K. (2001). "Nash Equilibrium when Players Account for the Complexity of their Forecast." Games and Economic Behavior, forthcoming.

5. Kalai E. and W. Stanford (1988). "Finite Rationality and Interpersonal Complexity in Repeated Games." Econometrica 56, 397-410.

6. Maenner E. (2001). "Learning to be Simple: Adaptation and Complexity in the Repeated Prisoner's Dilemma." Mimeo, PSU.

7. Piccione M. (1992). "Finite Automata Equilibria with Discounting." Journal of Economic Theory 56, 180-193.

8. Piccione M. and A. Rubinstein (1993). "Finite Automata Play a Repeated Extensive Game." Journal of Economic Theory 61, 160-168.

9. Rubinstein A. (1986). "Finite Automata Play the Repeated Prisoner's Dilemma." Journal of Economic Theory 39, 83-96.

10. Spiegler R. (2002). "Equilibrium in Justifiable Strategies: A Model of Reason-Based Choice in Extensive-Form Games." Review of Economic Studies, forthcoming.

\section{A ppendix: Proofs}

Define $q$ as a "stalemate" if $f(q)=D$ and $\tau(q, D)=q$. We will say that action $a$ is played against a state $q \in Q_{\mathrm{i}}$ if there exists a period $t$ along $z\left(s_{1}, s_{2}\right)$, such that $p_{\mathrm{i}}(t-1)=q$ and $a^{\mathrm{t}}=a$. Let $A_{\mathrm{j}}(q)$ denote the set of actions that 
player $j$ plays against $q \in Q_{\mathrm{i}}$. Note that the proofs of Propositions 3 and 4 rely on the proof of Proposition 2.

\subsection{Proposition 2}

The proof proceeds step-wise. Suppose that $\left(s_{1}, s_{2}\right)$ is a cyclic SINE satisfying the NRT property.

Claim 1 Each player plays both $C$ and $D$ in equilibrium.

P roof. If player $i$ plays only $D$ in $z\left(s_{1}, s_{2}\right)$, then the play path does not induce an interior agreement, a contradiction. If player $i$ plays only $C$, then a machine consisting of a single $C$-state is consistent with his behavior. This is the unique simplest machine for player $i$. Against this machine, the optimal response for player $j$ is to play always $D$. This does not induce an interior agreement, a contradiction.

Claim 2 For each player $i$, every state $q \in Q_{\mathrm{i}}$ is visited in $z\left(s_{1}, s_{2}\right)$.

P roof. Assume the contrary - i.e., that $q \in Q_{\mathrm{i}}$ is not visited in $z\left(s_{1}, s_{2}\right)$ for some player $i$. Then, $s_{\mathrm{i}} \notin B_{\mathrm{i}}\left(s_{1}, s_{2}\right)$ : an alternative machine $s_{\mathrm{i}}^{\prime}$, satisfying $Q_{\mathrm{i}}^{\prime}=Q_{\mathrm{i}} \backslash\{q\}$ and $\tau_{\mathrm{i}}^{\prime}(r, a) \in Q_{\mathrm{i}}^{\prime}$ whenever $\tau_{\mathrm{i}}(r, a)=q$, is simpler than $s_{\mathrm{i}}$ and consistent with $z\left(s_{1}, s_{2}\right)$.

Claim 3 If $q \in Q_{\mathrm{i}}$ is not a stalemate, then $A_{\mathrm{j}}(q)$ is a singleton.

P roof. Assume the contrary - i.e., $q \in Q_{\mathrm{i}}$ is not a stalemate and yet, there exist two periods $t, k$ in $z\left(s_{1}, s_{2}\right)$, such that $p_{\mathrm{i}}(t)=p_{\mathrm{i}}(k)=q$ and $a_{\mathrm{j}}^{\mathrm{t}+1}=C, a_{\mathrm{j}}^{\mathrm{k}+1}=D$. Let $t^{\prime}>t$ be the earliest period after $t$, in which $q$ is visited again. Similarly, let $k^{\prime}>k$ be the earliest period after $k$, in which $q$ is visited again. Consider the time interval between $t$ and $t^{\prime}$, and compare it to the time interval between $k$ and $k^{\prime}$.

Suppose that in both time intervals, at least one player plays $C$. Recall that the play path is cyclic. Therefore, if the concession ratios in the two time intervals are different, then player $j$ does not optimize against $s_{\mathrm{i}}$, a contradiction. On the other hand, if the concession ratios in the two time intervals are identical, then by the NRT property, $\tau_{\mathrm{i}}(q, C)=\tau_{\mathrm{i}}(q, D)$. But in this case, playing $C$ against $i$ is inconsistent with best-replying to $s_{\mathrm{i}}$. (It 
could be consistent with best-replying if $\left(s_{1}, s_{2}\right)$ sustained the limit concession ratio $x_{\mathrm{i}}=0$. However, recall that we are focusing on interior agreements.)

It follows that in the time interval between $k$ and $k^{\prime}$, neither player plays $C$. Since $q$ is not a stalemate, there exists a $D$-state $r \in Q_{\mathrm{i}}, r \neq q$, which is visited in this time interval. The following machine $s_{\mathrm{i}}^{\prime}$ for player $i$ is consistent with $z\left(s_{1}, s_{2}\right): Q_{\mathrm{i}}^{\prime}=Q_{\mathrm{i}} \backslash\{q\} ; \tau_{\mathrm{i}}^{\prime}(r, D)=\tau_{\mathrm{i}}(q, D) ; \tau_{\mathrm{i}}^{\prime}\left(r^{\prime}, a\right)=r$ whenever $\tau_{\mathrm{i}}\left(r^{\prime}, a\right)=q$; otherwise, $s_{\mathrm{i}}^{\prime}$ is identical to $s_{\mathrm{i}}$. Since $s_{\mathrm{i}}^{\prime}$ is simpler than $s_{\mathrm{i}}, s_{\mathrm{i}} \notin B_{\mathrm{i}}\left(s_{1}, s_{2}\right)$, a contradiction.

Claim 4 If $f_{\mathrm{i}}(q)=f_{\mathrm{i}}(r)$ and $q$ and $r$ are not stalemates, then $A_{\mathrm{j}}(q)=A_{\mathrm{j}}(r)$.

P roof. Assume the contrary. By Claim 3, exactly one action $a$ is played against $q$ and exactly one action $b \neq a$ is played against $r$. Then, the following machine $s_{\mathrm{i}}^{\prime}$ for player $i$ is consistent with $z\left(s_{1}, s_{2}\right): Q_{\mathrm{i}}^{\prime}=Q_{\mathrm{i}} \backslash\{q\} ; \tau_{\mathrm{i}}^{\prime}(r, a)=$ $\tau_{\mathrm{i}}(q, a) ; \tau_{\mathrm{i}}^{\prime}\left(r^{\prime}, a^{\prime}\right)=r$ whenever $\tau_{\mathrm{i}}\left(r^{\prime}, a^{\prime}\right)=q$; otherwise, $s_{\mathrm{i}}^{\prime}$ is identical to $s_{\mathrm{i}}$. Since $s_{\mathrm{i}}^{\prime}$ is simpler than $s_{\mathrm{i}}, s_{\mathrm{i}} \notin B_{\mathrm{i}}\left(s_{1}, s_{2}\right)$, a contradiction.

Claim 5 For every player $i$, there exists a stalemate $q \in Q_{\mathrm{i}}$.

P roof. Assume the contrary - i.e., that for some player $i, Q_{\mathrm{i}}$ does not include a stalemate. By Claim 4, player $j$ plays exactly one action $b$ against all $D$-states in $Q_{\mathrm{i}}$. By Claim 3, player $j$ plays exactly one action $a$ against all $C$-states in $Q_{\mathrm{i}}$. By Claim $1, a \neq b$. If $a=D$ and $b=C$, then it can easily be checked that player $i$ fails to play exactly one action against all $C$-states in $Q_{\mathbf{j}}$, in contradiction to Claim 3. On the other hand, if $a=C$ and $b=D$, then every $C$-state in $Q_{\mathrm{i}}$ which is visited in $z\left(s_{1}, s_{2}\right)$ can be reached from every $D$-state in $Q_{\mathrm{i}}$ which is visited in $z\left(s_{1}, s_{2}\right)$. By Claim 2 , every state in $Q_{\mathrm{i}}$ is visited in $z\left(s_{1}, s_{2}\right)$. It follows that if player $j$ 's strategy were to play always $D$, he would be able to enforce the outcome $x_{\mathrm{j}}=1$, in contradiction to the optimality of $s_{\mathrm{j}}$ against $s_{\mathrm{i}}$. Thus, there exists a $D$-state $q \in Q_{\mathrm{i}}$, such that player $A_{\mathrm{j}}(q)=\{C, D\}$. By Claim $3, q$ is a stalemate.

Claim 6 Let $q \in Q_{\mathrm{i}}$ be a stalemate. Let $r \in Q_{\mathrm{i}}$ be a D-state, such that $\tau_{\mathrm{i}}(r, D)=q$. Then, $D \notin A_{\mathrm{j}}(r)$.

P roof. Assume the contrary. By definition, $r$ is not a stalemate. By Claim $3, A_{\mathrm{j}}(r)=\{D\}$. Therefore, $s_{\mathrm{i}} \notin B_{\mathrm{i}}\left(s_{1}, s_{2}\right)$ : the following machine $s_{\mathrm{i}}^{\prime}$ for player $i$ is simpler than $s_{\mathrm{i}}$ and consistent with $z\left(s_{1}, s_{2}\right): Q_{\mathrm{i}}^{\prime}=Q_{\mathrm{i}} \backslash\{r\}$; $\tau_{\mathrm{i}}^{\prime}\left(r^{\prime}, a\right)=q$ whenever $\tau_{\mathrm{i}}\left(r^{\prime}, a\right)=r$; otherwise, $s_{\mathrm{i}}^{\prime}$ is identical to $s_{\mathrm{i}}$. 
Definition 6 A sequence of actions $\left(a^{\mathrm{k}}\right)_{\mathrm{k}=\mathrm{t}, \ldots, \mathrm{t}+\mathrm{K}}$ in $z\left(s_{1}, s_{2}\right), K \geq 2$, is called a $D$-sequence of length $K$ if $a^{\mathrm{k}}=D$ for every $k=t, \ldots, t+K-1$ and $a^{\mathrm{t}+\mathrm{K}}=C$. If $p(t) \in Q_{\mathrm{i}}$, we say that the $D$-sequence is begun by player $i$. If $p(t+K) \in Q_{\mathrm{i}}$, we say that the $D$-sequence is terminated by player $i$.

Claim 7 There exists a $D$-sequence in $z\left(s_{1}, s_{2}\right)$, which is begun and terminated by the same player.

P roof. Assume the contrary. By Claim $1, a^{\mathrm{t}}=C$ and $a^{\mathrm{t}+1}=D$ for some period $t$. Let $p(t) \in Q_{\mathrm{i}}$. By Claim 4, player $j$ plays only $D$ against all $C$-states in $Q_{\mathrm{i}}$. But this implies that player $i$ always plays $C$ and player $j$ always plays $D$ in $z\left(s_{1}, s_{2}\right)$, in contradiction to Claim 1 . This establishes the existence of a $D$-sequence. If every $D$-sequence is begun and terminated by different players, then are a player $i$ and two periods $t, k$, such that $p(t), p(k) \in Q_{\mathrm{j}}$, $a^{\mathrm{t}}=a^{\mathrm{k}}=C$ and $a^{\mathrm{t}+1} \neq a^{\mathrm{k}+1}$ - that is, player $i$ fails to play exactly one action against all $C$-states of his opponent's machine, contradicting Claim 4 .

Claim $8 s_{\mathrm{i}} \notin B_{\mathrm{i}}\left(s_{1}, s_{2}\right)$ for some player $i$.

Proof. by Claim 7 , there exists a $D$-sequence in $z\left(s_{1}, s_{2}\right)$. Pick the longest $D$-sequence and denote its length by $K^{*}$. (If there are several $D$ sequences of maximal length, pick one arbitrarily.) The $D$-sequence is terminated by some player $i$. Let $P \subset Q_{\mathrm{i}}$ be the set of $D$-states in $Q_{\mathrm{i}}$, which are visited during the $D$-sequence, and let $p^{0} \in P$ be the earliest state in $P$ to be visited in the sequence. By construction, none of the states in $P$ are stalemates. By Claim 4, player $j$ plays only $D$ against the states in $P$.

Let us turn to the $D$-sequences terminated by player $j, i$ 's opponent. There are two cases to consider.

Case 1. Either there exist no such sequences, or there exist such sequences but their maximal length is strictly below $\max \left(3, K^{*}\right)$. In this case, pick an arbitrary stalemate $q \in Q_{\mathrm{i}}$. (Such a state exists, by Claim 5).

Case 2. There exist $D$-sequences, which are terminated by player $j$, all of which are of length $K \leq K^{*}$, and some of which are exactly of length $K^{*}$ (where $K^{*} \geq 3$ ). by Claim 7 , there is a $D$-sequence, which is begun and terminated by the same player, say player $j$. Note that during this $D$ sequence, player $j$ plays both $C$ and $D$ against $D$-states in player $i$ 's machine. By Claims 4 and 6, player $i$ is in a stalemate $q$ during the entire $D$-sequence. 
In both cases, the following machine $s_{\mathrm{i}}^{\prime}$ is consistent with $z\left(s_{1}, s_{2}\right): Q_{\mathrm{i}}^{\prime}=$ $Q_{\mathrm{i}} \backslash\{q\} ; \tau_{\mathrm{i}}^{\prime}\left(q^{\prime}, a\right)=p^{0}$ whenever $\tau_{\mathrm{i}}\left(q^{\prime}, a\right)=q ; \tau_{\mathrm{i}}^{\prime}(p, C)=p^{0}$; otherwise, $s_{\mathrm{i}}^{\prime}$ is identical to $s_{\mathrm{i}}$. Since $s_{\mathrm{i}}^{\prime}$ is simpler than $s_{\mathrm{i}}, s_{\mathrm{i}} \notin B_{\mathrm{i}}\left(s_{1}, s_{2}\right)$.

\subsection{P roposition 3}

Let $\left(s_{1}, s_{2}\right)$ be a cyclic SINE, which sustains an interior agreement. By Proposition $2,\left(s_{1}, s_{2}\right)$ violates the NRT property. It is easy to show that $\left|Q_{\mathrm{i}}\right|>2$ for every $i=1,2$. (The proof is omitted.)

Suppose that there exists no $s_{\mathrm{i}}^{\prime}$ that satisfies the three conditions of the proposition. Claim 3 is the only part in the proof of Proposition 2 that employs NRT. Hence, whenever we can show that Claim 3 holds despite the violation of NRT, we can appropriate any other step in the proof of Proposition 2. Suppose that $A_{\mathrm{j}}(q)$ is a singleton for every $q \in Q_{\mathrm{i}}$ satisfying $V_{\mathrm{j}}(q, C)=V_{\mathrm{j}}(q, D)$. Note that $q$ is not a stalemate - otherwise, $V_{\mathrm{j}}(q, D)=0$. Thus, Claim 3 holds and the proof of Proposition 2 can be applied here to show that $\left(s_{1}, s_{2}\right)$ is not a SINE. Therefore, $A_{\mathrm{j}}(q)=\{C, D\}$ for some state $q \in Q_{\mathrm{i}}$ satisfying $\tau_{\mathrm{i}}(q, C) \neq \tau_{\mathrm{i}}(q, D)$ and $V_{\mathrm{j}}(q, C)=V_{\mathrm{j}}(q, D)$. (Hence, $q$ is not a stalemate.)

Since $\left(s_{1}, s_{2}\right)$ is cyclic and every state in $Q_{\mathrm{i}}$ is visited in $z\left(s_{1}, s_{2}\right)$, every two states $q, r \in Q_{\mathrm{i}}$ can be reached from each other. Order the states in $Q_{\mathrm{i}}$ as follows: $Q_{\mathrm{i}}=\left\{q^{1}, \ldots, q^{\mathrm{n}}\right\}, n=\left|Q_{\mathrm{i}}\right|$, such that for every $k=1, \ldots, n$ there exists an action $a^{\mathrm{k}} \in A_{\mathrm{j}}(q)$, such that $\tau_{\mathrm{i}}\left(q^{\mathrm{k}}, a^{\mathrm{k}}\right)=q^{\mathrm{k}+1 \operatorname{modn}}$. As we saw earlier, $Q_{\mathrm{i}}$ contains a state $q^{\mathrm{k}}$ which is not a stalemate, for which $A_{\mathrm{j}}\left(q^{\mathrm{k}}\right)=\{C, D\}$. Thus, there exist an action $b^{\mathrm{k}} \neq a^{\mathrm{k}}$ and another state $q^{\mathrm{k}+\mathrm{m}(\mathrm{k})} \in Q_{\mathrm{i}}, m(k) \neq 1$, such that $q^{\mathrm{k}+\mathrm{m}(\mathrm{k})}=\tau_{\mathrm{i}}\left(q^{\mathrm{k}}, b^{\mathrm{k}}\right)$. Let $Q^{\mathrm{K}} \subset Q_{\mathrm{i}}$ be the set of all such states $q^{\mathrm{k}}$. Denote $Q^{\mathrm{L}}=Q_{\mathrm{i}}-Q^{\mathrm{K}}$.

Suppose that player $j$ deviates to a machine $s_{\mathrm{j}}^{\prime}$, which plays only $b^{\mathrm{k}}$ against every state $q^{\mathrm{K}} \in Q^{\mathrm{K}}$ and only $a^{\mathrm{l}}$ against every state $q^{\mathrm{l}} \in Q^{\mathrm{L}}$. If, as a consequence of this deviation, a subset $Q^{*} \subset Q_{\mathrm{i}}$ consisting of at least two states becomes unvisited in $z\left(s_{\mathrm{i}}, s_{\mathrm{j}}^{\prime}\right)$, then the following machine $s_{\mathrm{i}}^{\prime}$ is simpler than $s_{\mathrm{i}}$ and consistent with $z\left(s_{\mathrm{i}}, s_{\mathrm{j}}^{\prime}\right): Q_{\mathrm{i}}^{\prime}=\left(Q_{\mathrm{i}}-Q^{*}\right) \cup\left\{q^{*}\right\}, f_{\mathrm{i}}\left(q^{*}\right)=D$, $\tau_{\mathrm{i}}^{\prime}\left(q^{*}, \cdot\right)=q^{*}, \tau_{\mathrm{i}}^{\prime}\left(q^{\mathrm{k}}, a^{\mathrm{k}}\right)=q^{*}$ for every $q^{\mathrm{k}} \in Q^{\mathrm{K}}, \tau_{\mathrm{i}}^{\prime}\left(q^{\mathrm{l}}, a\right)=q^{*}$ when $a \neq a^{\mathrm{l}}$ for every $q^{l} \in Q^{l}$; and otherwise $s_{\mathrm{i}}^{\prime}$ is identical to $s_{\mathrm{i}}$. In other words, $s_{\mathrm{i}}^{\prime}$ substitutes a single "grim" state for the states that become unvisited as a result of the deviation. (For example, suppose that the structure of $s_{\mathrm{j}}$ is given by Figure 6 and that $D \in A_{\mathrm{i}}(q)$. If player $i$ deviates to a strategy that always plays $C$ against $q$, he renders three states in $Q_{\mathrm{j}}$ unvisited. Therefore, 
his justification of his behavior can rely on an alternative belief $s_{\mathrm{j}}^{\prime}$, which substitutes a single "grim" state for these three states.) Note that $s_{\mathrm{j}}^{\prime}$ is a best-reply to $s_{\mathbf{i}}^{\prime}$. Hence, the deviation to $s_{\mathrm{j}}^{\prime}$ satisfies the three conditions of the proposition, a contradiction.

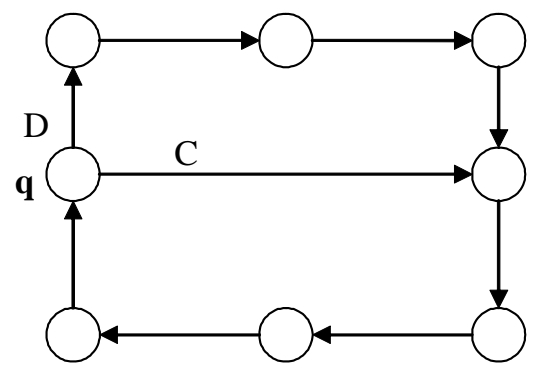

Figure 6

It follows that $Q^{\mathrm{K}}$ consists of a single element $q^{\mathrm{K}}$, such that $m(k)=2$. (Since $n>2, m(k) \bmod n \neq k$.) This also means that $q^{\mathrm{k}}$ is the only state that $q^{\mathrm{k}+1}$ is connected to - i.e., there exists no other state $q^{\mathrm{h}} \in Q_{\mathrm{i}}$, for which $\tau_{\mathrm{i}}\left(q^{\mathrm{h}}, a\right)=q^{\mathrm{k}+1}$ for some action $a \in\{C, D\}$.

Suppose that $a^{k}=C, b^{k}=D$. Consider the deviation of player $j$ to a machine $s_{\mathrm{j}}^{\prime}$, which plays only $D$ against $q^{\mathrm{k}}$ and only $a^{1}$ against every other state $q^{\prime}$. By construction, $s_{\mathrm{j}}^{\prime}$ is a best-reply to $s_{\mathrm{i}}$. Moreover, the following machine $s_{\mathrm{i}}^{\prime}$ is simpler than $s_{\mathrm{i}}$ and consistent with $z\left(s_{\mathrm{i}}, s_{\mathrm{j}}^{\prime}\right): Q_{\mathrm{i}}^{\prime}=$ $\left\{q^{1}, \ldots, q^{\mathrm{k}}, q^{\mathrm{k}+2}, \ldots, q^{\mathrm{n}}\right\} ; \tau_{\mathrm{i}}^{\prime}\left(q^{\mathrm{k}}, a\right)=q^{\mathrm{k}+2}$ for every $a \in\{C, D\}$; and otherwise, $s_{\mathrm{i}}^{\prime}$ is identical to $s_{\mathrm{i}}$. Note that $s_{\mathrm{j}}^{\prime}$ is a best-reply to $s_{\mathrm{i}}^{\prime}$. Hence, the deviation to $s_{\mathrm{j}}^{\prime}$ satisfies the three conditions of the proposition, a contradiction. It follows that $a^{\mathrm{k}}=D, b^{\mathrm{k}}=C$.

Claim $9 q^{\mathrm{k}+1}$ is a stalemate and $f_{\mathrm{i}}\left(q^{\mathrm{k}}\right)=C$.

P roof. Suppose that $a^{k+1}=D$. Then, it is possible for player $j$ to make player $i$ 's machine switch from $q^{\mathrm{k}}$ to $q^{\mathrm{k}+2}$ by playing only $D$ against $q^{\mathrm{k}}$ and $q^{\mathrm{k}+1}$, in contradiction to the optimality of playing $C$ against $q^{\mathrm{k}}$. (Recall that $V_{\mathrm{j}}\left(q^{\mathrm{k}}, a^{\mathrm{k}}\right)=V_{\mathrm{j}}\left(q^{\mathrm{k}}, b^{\mathrm{k}}\right)$. Thus, $a^{\mathrm{k}+1}=C$. Now, suppose that $f_{\mathrm{i}}\left(q^{\mathrm{k}+1}\right)=C$. If player $j$ plays $C$ against $q^{\mathrm{k}}$, player $i$ 's machine switches immediately to $q^{\mathrm{k}+2}$. 
On the other hand, if player $j$ plays $D$ against $q^{\mathrm{k}}$, he can do no better than play $C$ against $q^{\mathrm{k}+1}$ (since $a^{\mathrm{k}+1}=C$ ), and player $i$ 's machine will switch to $q^{\mathrm{k}+2}$. In both cases player $j$ makes one concession during the move from $q^{\mathrm{k}}$ to $q^{\mathrm{k}+2}$, but in the latter case he also manages to squeeze a concession from player $i$. Therefore, $V_{\mathrm{j}}\left(q^{\mathrm{k}}, D\right)>V_{\mathrm{j}}\left(q^{\mathrm{k}}, C\right)$, a contradiction. Thus, we have established that $a^{\mathrm{k}+1}=C$ and $f_{\mathrm{i}}\left(q^{\mathrm{k}+1}\right)=D$.

We have already shown that $q^{\mathrm{k}}$ is the only state $q \in Q_{\mathrm{i}}$ which is not a stalemate, for which $\tau_{\mathrm{i}}(q, C) \neq \tau_{\mathrm{i}}(q, D)$. If $\tau_{\mathrm{i}}\left(q^{\mathrm{k}+1}, D\right)=\tau_{\mathrm{i}}\left(q^{\mathrm{k}+1}, C\right)=q^{\mathrm{k}+2}$, then $C \notin \arg \max V\left(q^{\mathrm{k}+1}, \cdot\right)$, contradicting the fact that $a^{\mathrm{k}+1}=C$. Thus, the only remaining possibility is that $q^{\mathrm{k}+1}$ is a stalemate.

Suppose that $f_{\mathrm{i}}\left(q^{\mathrm{k}}\right)=D$. Then, the following machine $s_{\mathrm{i}}^{\prime}$ for player $i$ is consistent with $z\left(s_{1}, s_{2}\right): Q_{\mathrm{i}}^{\prime}=Q_{\mathrm{i}}-\left\{q^{\mathrm{k}}\right\} ; \tau_{\mathrm{i}}^{\prime}(r, a)=q^{\mathrm{k}+1}$ whenever $\tau_{\mathrm{i}}(r, a)=q^{\mathrm{k}} ; \tau_{\mathrm{i}}^{\prime}\left(q^{\mathrm{k}+1}, D\right)=q^{\mathrm{k}+1}$; otherwise, $s_{\mathrm{i}}^{\prime}$ is identical to $s_{\mathrm{i}}$. Therefore, $s_{\mathrm{i}} \notin B_{\mathrm{i}}\left(s_{1}, s_{2}\right)$, a contradiction.

Let us summarize what we have established so far. If there exists a state $q^{\mathrm{k}} \in Q_{\mathrm{i}}$ which is not a stalemate such that $A_{\mathrm{j}}\left(q^{\mathrm{k}}\right)=\{C, D\}$, then: $f_{\mathrm{i}}\left(q^{\mathrm{k}}\right)=C ; q^{\mathrm{k}+1}$ is a stalemate; $a^{\mathrm{k}}=D, a^{\mathrm{k}+1}=C ; q^{\mathrm{k}}$ is the only state from which there is a transition into $q^{\mathrm{k}+1}$; and $q^{\mathrm{k}}$ is the only state in $Q_{\mathrm{i}}$ that has a non-constant yet is not a stalemate.

Claim 10 Each player's machine contains a stalemate.

P roof. Suppose that $Q_{\mathrm{i}}$ does not contain a stalemate. Then, $Q_{\mathrm{i}}$ must contain a cycle of $D$-states: i.e., a subset of states $\bar{Q}$, through which player $i$ 's machine would cycle if player $j$ played only $D$ against them. Otherwise, player $j$ would be able to play only $D$ against $s_{\mathrm{i}}$ and attain $x_{\mathrm{j}}=1$, a contradiction. Recall the way in which we ordered the states in $Q$ from $q^{1}$ through $q^{\mathrm{n}}$. Note that $q^{\mathrm{l}} \notin \bar{Q}$. There must exist a state $q^{\mathrm{l}} \in \bar{Q}$, for which $a^{\prime}=C$ and $b^{\mid}=D$ - otherwise, the play path would not sustain an interior agreement. But this contradicts our previous assertion that $q^{\mathrm{k}}$ is the only state in $Q_{\mathrm{i}}$ that has a non-constant transition yet is not a stalemate.

Claim 11 There is a D-sequence that is begun and terminated by the same player.

P roof. We saw earlier that $q^{\mathrm{k}+1}$ is connected only to $q^{\mathrm{k}}$. Since $q^{\mathrm{k}}$ is visited in $z\left(s_{1}, s_{2}\right)$, there exists a period $t$, such that $p(t)=q^{\mathrm{k}}$ and $p(t+2)=q^{\mathrm{k}+1}$. 
Because $q^{\mathrm{k}+1}$ is a stalemate, player $j$ eventually plays $C$ against $q^{\mathrm{k}+1}$ at some period $t^{\prime}>t+2$. Thus, there exists a $D$-sequence, which is begun and terminated by player $j$.

Claim 12 Players play exactly one action against any D-state which is not a stalemate.

P roof. We have seen that if $q \in Q_{\mathrm{i}}$ is not a stalemate and player $j$ plays both $C$ and $D$ against $q$, then $q$ is a $C$-state.

The last three claims allow us to apply Claim 8 in the proof of Proposition 2. It follows that $\left(s_{1}, s_{2}\right)$ is not a SINE, a contradiction.

\subsection{Proposition 4}

Let $\left(s_{1}, s_{2}\right)$ be a SINE with linear machines, which sustains an interior agreement. It is easy to see that Claims 1 and 2 hold, irrespective of whether the concession game's horizon is finite or infinite. Let us now show that $A_{\mathrm{j}}(q)$ is a singleton whenever $q$ is not a stalemate. This would reproduce Claim 3 for the finite-horizon game with linear equilibrium machines, and we would be able to appropriate Claims $4-8$ as well.

Suppose that $A_{\mathrm{j}}(q)=\{C, D\}$ against some state $q \in Q_{\mathrm{i}}$, which is not a stalemate. For every player $i$, the last state $r_{\mathrm{i}}$ to be visited in $z\left(s_{1}, s_{2}\right)$ satisfies $\tau_{\mathrm{j}}\left(r_{\mathrm{i}}, \cdot\right)=r_{\mathrm{i}}$. Otherwise, by the linearity of equilibrium machines, there exists a state in one of the player's machines, which is not visited in $z\left(s_{1}, s_{2}\right)$, in contradiction to Claim 2. W.l.o.g., $f_{1}\left(r_{1}\right)=C$ and $f_{2}\left(r_{2}\right)=D$.

Suppose that player 2 deviated to a strategy that plays only $D$ against any state in $Q_{1}$ which is not a stalemate, and plays $C$ against any stalemate in $Q_{1}$. Due to the linearity of $s_{1}$, the deviation would strictly reduce the total number of concessions that player 1 makes. Since player 1's machine has an absorbing $C$-state which is reached as a result of the deviation, the game will terminate in agreement. Therefore, this is a profitable deviation

for player 2, a contradiction. We have thus established Claim 3. The rest of the proof of Proposition 2 can be applied. 